\title{
Prevalencia y tipos de maltrato en el noviazgo en adolescentes y adultos jóvenes
}

\author{
Prevalence and types of dating violence in adolescents and young adults
}

\author{
César Armando Rey-Anacona \\ Grupo de Investigación en Psicología Clínica y de la Salud \\ Universidad Pedagógica y Tecnológica de Colombia
}

(Rec: 17 de mayo de 2011 / Acept: 18 de enero de 2013)

\begin{abstract}
Resumen
Se examinó la prevalencia general y por sexo de alguna conducta de maltrato en el noviazgo, de tipo físico, psicológico, emocional, sexual, económico y negligente, en una muestra de 902 adolescentes y jóvenes adultos solteros colombianos, 417 varones y 485 mujeres, entre 15 y 35 años de edad. El 85.6\% informó haber sido objeto, por lo menos una vez, de alguna forma de maltrato por parte de su pareja, no encontrándose una diferencia estadísticamente significativa entre el porcentaje de varones y de mujeres que reportaron dicho maltrato. El tipo más frecuente fue el psicológico, seguido por el físico, el emocional, el sexual, el económico y el negligente. El porcentaje de varones que informó al menos una conducta de maltrato emocional, sexual, económico y negligente fue significativamente mayor que el de las mujeres, no hallándose diferencias estadísticamente significativas por sexo con respecto al maltrato físico y psicológico.

Palabras clave: Violencia de pareja, prevalencia, adolescentes, adultos jóvenes.
\end{abstract}

\begin{abstract}
General and sex-differences in prevalence were examined with regard to physical, psychological, emotional, sexual, economical, and negligent dating violence behaviors, in 902 adolescents and youths Colombian single adults, 417 males and 485 women, 15-35 years years-old. 85.6\% informed to have been object, at least once, of some abusive behavior. Not statistical differences by sex were found in this general prevalence. The more frequent maltreatment type was the psychological, continued by physical, emotional, sexual, economical, and negligent. Males informed more frequently some emotional, sexual, economical, and negligent abusive behavior than women. Not statistical differences by sex were found with regard to the physical and psychological abuse.

Key words: Partner violence, prevalence, adolescents, young adults.
\end{abstract}

\section{Introducción}

Los resultados de los estudios que se han realizado en los últimos años sobre la prevalencia de la violencia en las parejas jóvenes no casadas o que no conviven, indican que esta forma de violencia de pareja debería recibir tanta atención como la violencia en las parejas casadas, no solo por las cifras que se han reportado sino por sus potenciales efectos negativos en la salud física y mental de las víctimas (Centers for Disease Control and Prevention, 2006; Corral, 2009; Howard y Wang, 2003; Lewis y Fremouw, 2001; Matud, 2007; Muñoz-Rivas, Graña, O’Leary y González, 2007; Silverman, Raj, Mucci y Hathaway, 2001). Los estudios de prevalencia realizados en Estados Unidos han informado cifras de hasta el 88\% (Cornelius y Resseguie, 2007), que muestran que esta forma de violencia se encuentra

\footnotetext{
Nota del autor: Este proyecto fue aprobado y recibió apoyo financiero de la Dirección de Investigaciones de la Universidad Pedagógica y Tecnológica de Colombia, código SGI-458.

El autor agradece a las siguientes personas por su colaboración en esta investigación: Lizzet Paitán, Lorena Monguí, Maritza Mateus, Nidia Otálora, Paola Bayona, Pedro Mojíca, Rosalba Mariño, Arturo Martínez, Ibeth Guerrero, Sara Martínez, Alejandra Sosa, Víctor Rodríguez, Yeffer Roncancio, Jenny López, Jennifer Ramírez, Jenniffer Pineda, María Paula Pulido, Michael Pita, Fredy Acero, Mónica Bayona, Tatiana Castellanos y Yesica Áriza.

Correspondencia: Universidad Pedagógica y Tecnológica de Colombia, Facultad de Ciencias de la Salud, Escuela de Psicología, Calle 24 No. 5-63, Antiguo Hospital San Rafael, Tunja, Colombia. Correo electrónico: cesar.rey@uptc.edu.co.
} 
muy extendida entre adolescentes y adultos jóvenes y las problemáticas que se han relacionado con la misma incluyen el abuso de sustancias, las conductas sexuales riesgosas y los intentos de suicidio (Ackard y Neumark-Sztainer, 2002; Centers for Disease Control and Prevention, 2006; Rivera, Allen, Rodríguez, Chávez y Lazcano, 2007; Silverman et al., 2001).

En general, las investigaciones muestran que la violencia en el noviazgo tiende a caracterizarse por actos de maltrato menos graves comparados con aquellos que ocurren en la violencia marital y que no existen muchas diferencias por sexo en la prevalencia de los malos tratos físicos y psicológicos (Corral, 2009; Matud, 2007; Rey-Anacona, 2008a; Weisz, Tolman, Callahan, Saunders y Black, 2007). Los "Centros para la Prevención y el Control de la Enfermedad" de los Estados Unidos (Centers for Disease Control and Prevention, 2006), por ejemplo, encontraron que el $8.9 \%$ de los varones y el $8.8 \%$ de las mujeres habían sido objeto de violencia física por parte de su pareja, entre 15214 adolescentes de noveno a duodécimo grados, vinculados a escuelas públicas y privadas de 50 estados y el Distrito de Columbia. Sears, Byers y Price (2007), por su parte, informaron que el 35\%, 15\% y 17\% de los varones habían realizado actos de violencia psicológica, física y sexual, respectivamente, mientras que un 47\%, 28\% y 5\% de las mujeres llevaron a cabo actos de la misma naturaleza, respectivamente, entre 633 adolescentes canadienses de 12 a 18 años de edad, datos que señalan incluso que las mujeres podrían ejecutar con mayor frecuencia actos de violencia física, aunque menos de violencia sexual comparadas con los varones.

Los estudios realizados en Iberoamerica también tienden a mostrar cifras altas y proporciones similares por género. Gonzáles y Santana (2001), por ejemplo, encontraron una prevalencia de $7.5 \%$ y $7.1 \%$ de varones y mujeres, respectivamente, que reportaron haber empujado o pegado a su pareja, entre 1146 estudiantes de 16 a 18 años de edad que habían tenido al menos una relación de pareja, oriundos de Santa Cruz de Tenerife (España). Muñoz-Rivas et al. (2007), por su parte, estudiaron la prevalencia de conductas agresivas de tipo verbal y físico, entre 2416 adolescentes solteros escolarizados de 16 a 20 años de edad (1416 mujeres y 1000 varones), oriundos de Madrid (España), que tenían al menos una relación heterosexual, encontrando una prevalencia de conductas verbales agresivas del $95.3 \%$ para las mujeres y $92.8 \%$ para los varones y una prevalencia de actos de agresión física del $2 \%$ entre las mujeres y del $4.6 \%$ entre los varones.

Rivera et al. (2007), igualmente, estudiaron la prevalencia de violencia física y psicológica entre 7960 estudiantes de varias escuelas públicas de la provincia de Morelos, México, entre 11 y 24 años, que habían tenido al menos una relación de pareja, hallando una prevalencia de violencia psicológica del $4.21 \%$ para las mujeres y del $4.33 \%$ entre los varones, así como una prevalencia de violencia física del $20.99 \%$ para las mujeres y del $19.54 \%$ para los varones. Asimismo, encontraron que el $9.37 \%$ de las mujeres y el $8.57 \%$ de los varones habían sido víctimas de violencia psicológica, mientras que $9.88 \%$ de las mujeres y el $22.71 \%$ de los varones fueron víctimas de violencia física.

Si bien estas cifras parecen variar de un estudio a otro, dependiendo de los instrumentos utilizados y la definición operacional de violencia (Hanson, 2002; Lewis y Fremouw, 2001; Matud, 2007; Rivera, Allen, Rodríguez, Chávez y Lazcano, 2006), en general muestran que la forma de violencia en el noviazgo más frecuente es la psicológica, seguida por la física y la sexual, afectando esta última más a las mujeres que a los varones.

Dos formas de violencia de pareja que no se han estudiado suficientemente en relación con los malos tratos en el noviazgo son la violencia económica y la negligencia o descuido. La primera, según el Reglamento Técnico para el Abordaje Forense Integral de la Violencia Intrafamiliar de Pareja (Instituto Nacional de Medicina Legal y Ciencias Forenses, 2005), se presenta cuando se controla el acceso, manejo y gasto del dinero por parte de la pareja, mientras que Stordeur y Stille (1989), la conceptualizan como una forma de violencia de pareja en donde a ésta no se le deja trabajar cuando puede hacerlo y la otra persona aprovecha el hecho de que su pareja depende económicamente de ella. La Organización Mundial de la Salud (Krug, Dahlberg, Mercy, Zwi y Lozano, 2003), por su parte, considera que el descuido es una forma de violencia psíquica que puede presentarse con respecto a los menores, los ancianos y la pareja y en donde se presentan "actos por omisión", poniendo en riesgo a la víctima. La negligencia, en esa misma línea, es considerada por Pueyo, López y Álvarez (2008), como una forma pasiva de violencia de pareja, evidente especialmente en víctimas en desventaja física o social. Estas definiciones indican que estos tipos de maltrato también podrían presentarse en las relaciones de noviazgo, por lo que también deberían estudiarse para efectos de una más amplia descripción y comprensión del fenómeno.

Un estudio realizado con adolescentes y adultos jóvenes colombianos solteros víctimas de al menos un acto de maltrato por parte de su pareja (Rey-Anacona, 2009), encontró que la prevalencia general de algún tipo de maltrato era de $82.6 \%$, no existiendo diferencias estadísticamente significativas por sexo en cuanto a dicha prevalencia. En este trabajo también 
se examinó los malos tratos físicos, psicológicos, emocionales, sexuales y económicos, midiéndose estos últimos a través de ítems referentes a conductas como "Forzar a la otra persona a depender económicamente del agresor, no dejándola trabajar o por otros medios; ejercer control sobre los recursos financieros de la víctima o explotarla económicamente" (p. 30), encontrando una prevalencia por cada tipo de maltrato de $22.4 \%, 81.1 \%, 31.5 \%, 8.3 \%$ y $18.2 \%$, respectivamente, no observándose diferencias por sexo en cada uno de ellos, excepto en los malos tratos económicos, los cuales fueron más frecuentemente informados por los varones. Estos datos son coherentes, en general, con la literatura especializada internacional, pero indican, a diferencia de lo hallado previamente, que en el noviazgo también se pueden presentar malos tratos que pueden considerarse de índole económico, los cuales sufrirían más los varones que las mujeres. En un estudio posterior (Rey-Anacona, Mateus-Cubides y Bayona-Arévalo, 2010), se examinó la prevalencia de realización de alguna conducta de maltrato hacia la pareja, entre adolescentes de 15 a 20 años de edad, solteros y sin hijos, incluyendo los malos tratos de tipo físico, psicológico, emocional, sexual, económico y negligente, el último de los cuales se examinó a través de ítems referentes a conducta como no advertir a la pareja sobre algún peligro para su integridad física o psicológica. Los resultados revelaron que no existían diferencias significativas por sexo en cuanto a los malos tratos físicos y psicológicos, aunque si en los de tipo emocional, sexual y económico, los cuales fueron más frecuentemente ejercidos por los varones y que los malos tratos que podrían considerarse negligentes desde esta perspectiva también podrían presentarse en el noviazgo, siendo más frecuentemente ejecutados por los varones.

Con base en lo anterior, este trabajo se planteó como objetivo general examinar la prevalencia general y por sexo de alguna conducta de maltrato de tipo físico, psicológico, emocional, sexual, económico y negligente, realizada por una muestra de adolescentes y adultos jóvenes adultos colombianos, solteros y sin hijos, incluyendo la muestra de adolescentes estudiada por Rey-Anacona et al. (2010). De esta manera se pretendía identificar de manera más completa el fenómeno, examinado su prevalencia en adolescentes y adultos jóvenes colombianos y su prevalencia con respecto a los seis tipos de maltrato mencionados. Para ello, igualmente, delimitó como objetivos específicos: a) Determinar las diferencias por sexo en la frecuencia de realización de 82 conductas de maltrato hacia la pareja de los seis tipos señalados y b) examinar la correlación existente entre el tiempo de relación y la frecuencia de malos tratos, a nivel general y por cada tipo de maltrato.

\section{Método}

\section{Participantes}

En total participaron 902 estudiantes vinculados a 22 carreras profesionales y de licenciatura de una universidad pública colombiana, 417 varones $(46.2 \%)$ y 485 mujeres (53.8\%), entre 15 y 35 años de edad $(M=19.32$ años; $D T$ $=2.33$ años). Los participantes vivían en barrios de estrato socioeconómico bajo bajo $(\mathrm{n}=43,4.8 \%)$, bajo $(\mathrm{n}=312$, $34.6 \%)$, medio bajo $(\mathrm{n}=384,42.6 \%)$, medio $(\mathrm{n}=120$, $13.3 \%)$, medio alto $(n=20,2.2 \%)$ y alto $(n=2,0.2 \%)$ y se encontraban cursando entre el primer y el noveno semestres de estudios, con un promedio de 2.37 semestres cursados. Estos participantes fueron seleccionados de manera incidental, no probabilística, entre los cursos programados para los primeros cinco semestres de las carreras en mención, dado el interés de la investigación, escogiéndose aquellos que tuvieran o hubieran tenido una pareja y que fueran solteros y sin hijos.

\section{Instrumento}

Lista de Chequeo de Experiencias de Maltrato en la Pareja (Rey-Anacona, 2008b). Es un cuestionario de carácter descriptivo, no psicométrico, que permite informar la realización de 95 conductas de maltrato hacia la pareja, de tipo físico, verbal, emocional, psicológico, sexual, económico y negligente, por medio de una escala Likert con las siguientes opciones: "Nunca", "Una vez", "Algunas veces" o "Muchas veces". Este instrumento fue validado a nivel de contenido por jueces con titulación mínima de Magíster y experiencia investigativa en violencia intrafamiliar, quienes evaluaron la redacción de las instrucciones y los ítems y su pertinencia con respecto al tipo de violencia al que pertenecían. Rey-Anacona et al., (2010) informaron un alfa bastante alto (.96), que indica que el instrumento es muy consistente internamente, encontrando que el $50.54 \%$ de la varianza podría explicarse por seis factores, lo cual corresponde con el número de tipos de maltrato analizados. Los ítems de este cuestionario parten de las siguientes definiciones:

Maltrato físico. Cualquier acto dirigido al cuerpo de la persona, que produce daño o dolor sobre la misma (golpes, patadas, cachetadas, pellizcos, intento de estrangulamiento, etc.).

Maltrato psicológico. Cualquier acción dirigida a: a) controlar, restringir los movimientos o vigilar a la otra persona; b) aislarla socialmente; c) desvalorizarla, denigrarla, humillarla o hacerla sentir mal consigo misma; d) hacer que 
otros se pongan en su contra, acusarla falsamente o culparla por circunstancias negativas; e) obligarla a ir en contra de la ley o de sus creencias morales y/o religiosas o f) destruir su confianza en sí misma o en la pareja.

Maltrato emocional. Cualquier acto de naturaleza verbal o no verbal que provoca intencionalmente en la víctima una reacción de ansiedad, temor o miedo, como las intimidaciones $\mathrm{y}$ las amenazas; incluye los actos de violencia dirigidos a un familiar o a un conocido de la víctima, a sus bienes o hacia el agresor mismo, realizados con el mismo fin.

Maltrato sexual. Cualquier acto obligado, no consentido por la víctima, dirigido a satisfacer necesidades o deseos sexuales del victimario.

Maltrato económico. Forzar a la otra persona a depender económicamente del agresor, no dejándola trabajar o por otros medios; ejercer control sobre los recursos financieros de la víctima o explotarla económicamente.

Negligencia. No proporcionar (o no hacerlo debidamente) recursos financieros o materiales, información o servicios a la pareja, a pesar de que el agresor está obligado legalmente a hacerlo; no brindar ayuda económica o material a la pareja cuando ésta lo necesitaba y el victimario estaba en capacidad de hacerlo o no advertir a la pareja sobre algún peligro para su integridad física o psicológica (Rey-Anacona et al., 2010, p. 171).

Para efectos de este estudio, solamente se analizaron las respuestas a los 82 primeros ítems de este instrumento, ya que los 13 restantes se dirigen a personas que han conformado un hogar o ha tenido hijos con su pareja.

\section{Procedimiento}

El cuestionario fue administrado por estudiantes de estudios medios de psicología, quienes recibieron una capacitación teórica en los aspectos conceptuales y teóricos de la violencia de pareja y un entrenamiento en la aplicación del instrumento a través de una capacitación teórica y ensayos de administración. Dichos estudiantes también recibieron una capacitación en las normas éticas fundamentales contempladas en la investigación (Vg., anonimato, confidencialidad, consentimiento informado, libertad para retirarse voluntariamente e independencia de la investigación en relación con el proceso educativo), así como en un protocolo de información dirigido a la consecución del consentimiento informado de los participantes, que cada estudiante ensayó grupalmente.

Los participantes fueron contactados en su salón de clases, según la disponibilidad del caso y se les proporcionó la siguiente información para obtener su consentimiento informado: a) El procedimiento de la investigación y sus posibles molestias; b) la posibilidad de no aceptar participar en el estudio o de retirarse durante la aplicación de los instrumentos, sin que ello conllevase repercusiones; c) el anonimato y el mantenimiento reservado de los cuestionarios; d) la opción de ofrecer los resultados obtenidos al participante, si este los solicitase tras la recolección de los mismos y e) la independencia de la investigación con respecto a su proceso educativo. Debido a que el nivel de riesgo por participar en esta investigación era mínimo, a la luz de los lineamientos de la Resolución 008430 de 1993 (Ministerio de Salud de Colombia, 1993), no se consideró necesario solicitar dicho consentimiento informado por escrito sino de manera verbal.

En total, se aplicaron 1091 cuestionarios de forma colectiva en el mismo salón de clases, administración que fue supervisada por integrantes del grupo de investigación a cargo de la investigación. De estos 1091 se descartaron 189 ya que los participantes informaron que tenían hijos, no eran solteros o porque no habían tenido una relación de pareja. Los datos obtenidos, posteriormente, se incorporaron en una base de datos de SPSS versión 12.0 (SPSS, 2003) y se utilizó la prueba Chi-Cuadrada para comparar el número de participantes varones y mujeres que reportaron la ejecución de al menos una conducta de maltrato, tanto a nivel general como por cada tipo, para lo cual se codificó dicha realización con 1 y su ausencia con 0 . Igualmente, se utilizó el Análisis de Varianza Unidireccional para comparar por sexo la frecuencia de realización de las 82 conductas estudiadas, para lo cual se codificó dicha frecuencia de la siguiente manera: "Nunca" = 0 , "Una vez" = 1, "Algunas veces" $=2 \mathrm{y}$ "Muchas veces" $=3$. Asimismo, se implementó la formula de Pearson para evaluar la correlación existente entre el tiempo de relación y la frecuencia promedio de malos tratos, previamente codificada según lo que se indicó, a nivel general y por cada tipo de maltrato. Todos los análisis se hicieron aceptándose un nivel mínimo de probabilidad de .05., bilateral.

\section{Resultados}

El 85.6\% $(n=772)$ de los participantes reportó haber ejercido por lo menos una de las conductas que se presentan en el instrumento utilizado, de los cuales el $46.9 \%$ eran varones $(n=362)$ y el $53.1 \%$ mujeres $(n=410)$, no hallándose una diferencia estadísticamente significativa entre estos dos porcentajes $\left(X^{2}[1,902]=.94, p=.343\right)$. En relación con el porcentaje de participantes que informó al menos una 
conducta de cada tipo de maltrato, se encontró que el mayor fue el psicológico, seguido por el físico, el emocional, el sexual, el negligente y el económico (véase la Tabla 1).

Comparando estos porcentajes por sexo, se halló que el porcentaje de varones que informó al menos una conducta de maltrato emocional, sexual, económico y negligente fue significativamente mayor que el de las mujeres, no hallándose diferencias estadísticamente significativas con respecto a los malos tratos de tipo físico y psicológico (ver tabla $2 \mathrm{La}$ tabla de contingencia resultante entre el cruce de la edad y el número de participantes que informó haber ejercido al menos una conducta de maltrato, reveló que en todas las edades hubo al menos uno que informó esta situación, oscilando los porcentajes entre el $50 \%$ y el 100\% (se exceptúa el rango de 30 a 34 años, en el que no hubo participantes) -véase la Tabla 2-.
En la Tabla 3 se presenta la frecuencia promedio de realización de cada una de las 82 conductas de maltrato estudiadas, que se presentan en la lista de chequeo, señalándose las diferencias significativas por sexo. Tomando como punto de corte una desviación estándar por encima de la media general de malos tratos (Vg., 0.37) se puede observar que los participantes ejercieron con mayor frecuencia las siguientes conductas de maltrato: a) "La (lo) vigiló en su lugar de estudio, de trabajo o en otro espacio distinto"; b) "Controló su tiempo"; c) "Controló sus actividades cotidianas"; d) "Impuso su punto de vista sobre el suyo"; e) "Habló mal de ella (él) con amigos o conocidos"; f) "La (lo) acusó de ser infiel sin tener pruebas de ello"; g) "La (lo) llamó varias veces al día para averiguar que estaba haciendo" y h) "Se refirió a asuntos pasados para hacerla (lo) sentir mal".

Tabla 1. Porcentaje de participantes por sexo que ejercieron conductas de cada tipo de maltrato.

\begin{tabular}{|c|c|c|c|c|c|}
\hline Tipo de maltrato & Sexo & $\mathrm{n}$ & $\%$ & $\mathrm{X}^{2}$ & $\mathrm{p}$ \\
\hline \multirow[t]{3}{*}{ Físico } & Varones & 168 & 40.3 & 0.008 & .946 \\
\hline & Mujeres & 194 & 40 & & \\
\hline & Total & 362 & 40.1 & & \\
\hline \multirow[t]{3}{*}{ Psicológico } & Varones & 350 & 83.9 & 0.252 & .655 \\
\hline & Mujeres & 401 & 82.7 & & \\
\hline & Total & 751 & 83.3 & & \\
\hline \multirow[t]{3}{*}{ Emocional } & Varones & 183 & 43.9 & 13.129 & $.000^{* * *}$ \\
\hline & Mujeres & 156 & 32.2 & & \\
\hline & Total & 339 & 37.6 & & \\
\hline \multirow[t]{3}{*}{ Sexual } & Varones & 121 & 29 & 16.801 & $.000 * * *$ \\
\hline & Mujeres & 85 & 17.5 & & \\
\hline & Total & 206 & 22.8 & & \\
\hline \multirow[t]{3}{*}{ Económico } & Varones & 63 & 15.1 & 11.927 & $.001 * * *$ \\
\hline & Mujeres & 38 & 7.8 & & \\
\hline & Total & 101 & 11.2 & & \\
\hline \multirow[t]{3}{*}{ Negligente } & Varones & 85 & 20.4 & 18.735 & $.000 * * *$ \\
\hline & Mujeres & 49 & 10.1 & & \\
\hline & Total & 134 & 14.9 & & \\
\hline
\end{tabular}

Nota. $* * * p \leq .001$

Tabla 2. Número y porcentaje de participantes por edad que informaron al menos una conducta de maltrato $(N=902)$.

\begin{tabular}{lcccccccccccccccccc}
\hline Edad & 15 & 16 & 17 & 18 & 19 & 20 & 21 & 22 & 23 & 24 & 25 & 26 & 27 & 28 & 29 & 30 & 35 \\
\hline $\begin{array}{l}\text { Total } \\
\text { participantes }\end{array}$ & 4 & 30 & 140 & 220 & 169 & 129 & 81 & 46 & 29 & 23 & 12 & 7 & 2 & 6 & 1 & 2 & 1 \\
$\begin{array}{l}\text { Informaron } \\
\text { maltrato }\end{array}$ & 2 & 26 & 116 & 184 & 149 & 114 & 70 & 37 & 26 & 21 & 10 & 6 & 2 & 5 & 1 & 2 & 1 \\
\begin{tabular}{l}
$\%$ por edad \\
\hline
\end{tabular} & 50 & 86.7 & 82.9 & 84.5 & 87.6 & 89.1 & 85.2 & 80.4 & 93.1 & 91.3 & 83.3 & 85.7 & 100 & 83.3 & 100 & 50 & 100 \\
\hline
\end{tabular}


Los varones, específicamente, ejercieron con una frecuencia estadísticamente mayor las siguientes conductas de maltrato:

1. "La (lo) forzó a tener relaciones sexuales".

2. "Le robó dinero".

3. "La (lo) dejó sola (lo) en un lugar peligroso".

4. "Le prohibió comprar objetos para su uso personal".

5. "La (lo) amenazó con agredir a un miembro de su familia".

6. "La (lo) apretó fuerte con intención de lastimarla (lo)".

7. "La (lo) obligó a tener comportamientos sexuales que a ella (él) no le agradaban o con los que no se sentía a gusto".

8. "La (lo) puso en riesgo de contraer alguna enfermedad de transmisión sexual, a pesar de que sabía que ello podía ocurrir".

9. "Puso a su familia o a un miembro de su familia en su contra".

10. "Puso a la familia de ella (él) o a un miembro de dicha familia en su contra".

11. "Rompió o destruyó algún bien personal suyo".

12. "Utilizó elementos u objetos que podían ocasionar dolor a lesiones, durante una relación sexual".

13. "La (lo) presionó para que le cediera un bien personal, como un carro, una casa, un apartamento o una finca".

14. "La (lo) obligó a ser cómplice en situaciones ilegales".

15. "Se dirigió a ella (él) con gestos o palabras sexualmente degradantes".

16. "Le robó un objeto valioso o de valor sentimental para ella (él)".
17. "Le criticó su aspecto físico (por ejemplo, ser gorda(o), delgada(o), etc.)".

18. "Le dijo que iba a dañar bienes personales de un miembro de la familia de ella (él)".

19. "Le acarició una parte de su cuerpo de una manera vulgar, sin su consentimiento".

20. "Inventó que ella (él) le había robado algo".

21. "Llegó borracho(a) a su casa a hacer escándalo".

22. "Controló su tiempo".

23. "La (lo) amenazó con tener una relación con otra persona".

24. "La (lo) amenazó con hacerse daño a sí mismo(a), si ella (él) no hacía algo que usted había dicho".

25. "Le dijo que se avergonzaba de ella (él)".

26. "La (lo) amenazó con encerrarla (lo) en una institución mental".

27. "La (lo) obligó a consumir sustancias psicoactivas (por ejemplo, marihuana, cocaína, bazuco, etc.)".

28. "La (lo) amenazó con matar a un familiar de ella (él)".

29. " $\mathrm{La}$ (lo) persiguió cuando salió de la casa o de otro sitio".

30. "No la (lo) dejó entrar a la casa de ella (él)".

31. "La (lo) hizo sentirse mal, diciéndole que no lo (la) satisfacía sexualmente".

32. "Le echó en cara lo que usted le había brindado económicamente".

33. "Le puso sobrenombres para hacerla (lo) sentir mal".

Tabla 3. Diferencias por sexo en las conductas de maltrato ejercidas por los participantes

\begin{tabular}{|c|c|c|c|c|c|c|}
\hline \multirow{2}{*}{ Maltrato informado } & \multicolumn{4}{|c|}{$\mathrm{M}(\mathrm{DE})$} & \multirow[b]{2}{*}{$\mathrm{F}$} & \multirow[b]{2}{*}{$\mathrm{p}$} \\
\hline & Tipo de maltrato & Gral. & Var. & Muj. & & \\
\hline $\begin{array}{l}\text { 1. La (lo) vigiló en su lugar de estudio, de trabajo o en otro } \\
\text { espacio distinto. }\end{array}$ & Psicológico & $\begin{array}{c}0.40 \\
(0.71)\end{array}$ & $\begin{array}{c}0.42 \\
(0.72)\end{array}$ & $\begin{array}{c}0.39 \\
(0.71)\end{array}$ & 0.425 & .515 \\
\hline $\begin{array}{l}\text { 2. La (lo) amenazó con un objeto peligroso (por ejemplo, } \\
\text { un mazo, una piedra, etc.). }\end{array}$ & Emocional & $\begin{array}{c}0.06 \\
(0.28)\end{array}$ & $\begin{array}{c}0.06 \\
(0.29)\end{array}$ & $\begin{array}{c}0.06 \\
(0.27)\end{array}$ & 0.010 & .922 \\
\hline 3. Le arrojó algún objeto en un momento de conflicto. & Físico & $\begin{array}{c}0.12 \\
(0.39)\end{array}$ & $\begin{array}{c}0.09 \\
(0.35)\end{array}$ & $\begin{array}{c}0.14 \\
(0.42)\end{array}$ & 3.602 & .058 \\
\hline 4. La (lo) forzó a tener relaciones sexuales. & Sexual & $\begin{array}{c}0.06 \\
(0.28)\end{array}$ & $\begin{array}{c}0.09 \\
(0.35)\end{array}$ & $\begin{array}{c}0.04 \\
(0.20)\end{array}$ & 6.307 & $.012 *$ \\
\hline 5. Le robó dinero. & Económico & $\begin{array}{c}0.08 \\
(0.32)\end{array}$ & $\begin{array}{c}0.12 \\
(0.40)\end{array}$ & $\begin{array}{c}0.04 \\
(0.21)\end{array}$ & 14.597 & $.000 * * *$ \\
\hline 6. La (lo) dejó sola (lo) en un lugar peligroso. & Negligencia & $\begin{array}{c}0.16 \\
(0.43)\end{array}$ & $\begin{array}{c}0.21 \\
(0.50)\end{array}$ & $\begin{array}{c}0.11 \\
(0.37)\end{array}$ & 13.458 & $.000 * * *$ \\
\hline 7. Le prohibió comprar objetos para su uso personal. & Psicológico & $\begin{array}{c}0.10 \\
(0.39)\end{array}$ & $\begin{array}{c}0.13 \\
(0.44)\end{array}$ & $\begin{array}{c}0.07 \\
(0.34)\end{array}$ & 4.825 & $.028 *$ \\
\hline 8. La (lo) amenazó con agredir a un miembro de su familia. & Emocional & $\begin{array}{c}0.04 \\
(0.24)\end{array}$ & $\begin{array}{c}0.07 \\
(0.31)\end{array}$ & $\begin{array}{c}0.02 \\
(0.17)\end{array}$ & 6.813 & $.009 * *$ \\
\hline
\end{tabular}




Maltrato informado
9. La (lo) apretó fuerte con intención de lastimarla (lo).
10. La (lo) obligó a tener comportamientos sexuales que a
ella (él) no le agradaban o con los que no se sentía a gusto.
11. No la (lo) dejó trabajar o estudiar o la (lo) obligó a aban-
donar un trabajo o unos estudios que venía realizando.
12. La (lo) puso en riesgo de contraer alguna enfermedad
de transmisión sexual, a pesar de que sabía que ello
podía ocurrir.
13. Puso a su familia o a un miembro de su familia en su
contra.

14. La (lo) amenazó con el puño u otra parte del cuerpo.

15. La (lo) golpeó con una parte del cuerpo (con el puño, un pie, etc.).

16. La (lo) obligó a tener relaciones sexuales con otra persona.

17. Le exigió que le entregara el dinero que ella (él) se había ganado en su trabajo o por otro medio.

18. Puso a la familia de ella (él) o a un miembro de dicha familia en su contra.

19. Rompió o destruyó algún bien personal suyo.

20. Utilizó elementos u objetos que podían ocasionar dolor a lesiones, durante una relación sexual.

21. Se burló de sus deseos sexuales.

22. La (lo) presionó para que le cediera un bien personal, como un carro, una casa, un apartamento o una finca.

23. La (lo) obligó a ser cómplice en situaciones ilegales.

24. La (lo) amenazó con un cuchillo o cualquier otro elemento corto punzante.

25. Envió a alguien a hacerle daño a ella (él).

26. Se dirigió a ella (él) con gestos o palabras sexualmente degradantes.

27. Le robó un objeto valioso o de valor sentimental para ella (él).

28. Le criticó su aspecto físico (por ejemplo, ser gorda(o), delgada(o), etc.).

29. Le dijo que iba a dañar bienes personales de un miembro de la familia de ella (él).

\begin{tabular}{|c|c|c|c|c|c|}
\hline \multicolumn{4}{|c|}{$\mathrm{M}(\mathrm{DE})$} & \multirow[b]{2}{*}{$\mathrm{F}$} & \multirow[b]{2}{*}{$\mathrm{p}$} \\
\hline Tipo de maltrato & Gral. & Var. & Muj. & & \\
\hline Físico & $\begin{array}{c}0.21 \\
(0.52)\end{array}$ & $\begin{array}{c}0.25 \\
(0.55)\end{array}$ & $\begin{array}{c}0.17 \\
(0.49)\end{array}$ & 4.739 & $.030 *$ \\
\hline Sexual & $\begin{array}{c}0.08 \\
(0.33)\end{array}$ & $\begin{array}{c}0.13 \\
(0.44)\end{array}$ & $\begin{array}{c}0.04 \\
(0.19)\end{array}$ & 16.984 & $.000 * * *$ \\
\hline Económico & $\begin{array}{c}0.06 \\
(0.27)\end{array}$ & $\begin{array}{c}0.08 \\
(0.30)\end{array}$ & $\begin{array}{c}0.05 \\
(0.25)\end{array}$ & 1.584 & .208 \\
\hline Negligencia & $\begin{array}{c}0.05 \\
(0.27)\end{array}$ & $\begin{array}{c}0.08 \\
(0.35)\end{array}$ & $\begin{array}{c}0.03 \\
(0.17)\end{array}$ & 8.635 & $.003 * *$ \\
\hline Psicológico & $\begin{array}{c}0.18 \\
(0.51)\end{array}$ & $\begin{array}{c}0.24 \\
(0.59)\end{array}$ & $\begin{array}{c}0.14 \\
(0.43)\end{array}$ & 8.299 & $.004 * *$ \\
\hline Emocional & $\begin{array}{c}0.13 \\
(0.44)\end{array}$ & $\begin{array}{c}0.13 \\
(0.47)\end{array}$ & $\begin{array}{c}0.13 \\
(0.42)\end{array}$ & 0.084 & .772 \\
\hline Físico & $\begin{array}{c}0.15 \\
(0.48)\end{array}$ & $\begin{array}{c}0.13 \\
(0.48)\end{array}$ & $\begin{array}{c}0.16 \\
(0.48)\end{array}$ & 0.783 & .376 \\
\hline Sexual & $\begin{array}{c}0.05 \\
(0.21)\end{array}$ & $\begin{array}{c}0.06 \\
(0.24)\end{array}$ & $\begin{array}{c}0.04 \\
(0.19)\end{array}$ & 3.056 & .081 \\
\hline Económico & $\begin{array}{c}0.04 \\
(0.24)\end{array}$ & $\begin{array}{c}0.06 \\
(0.30)\end{array}$ & $\begin{array}{c}0.03 \\
(0.19)\end{array}$ & 1.796 & .181 \\
\hline Psicológico & $\begin{array}{c}0.12 \\
(0.40)\end{array}$ & $\begin{array}{c}0.17 \\
(0.48)\end{array}$ & $\begin{array}{c}0.07 \\
(0.31)\end{array}$ & 12.131 & $.001 * * *$ \\
\hline Emocional & $\begin{array}{c}0.19 \\
(0.46)\end{array}$ & $\begin{array}{c}0.25 \\
(0.54)\end{array}$ & $\begin{array}{c}0.14 \\
(0.38)\end{array}$ & 13.713 & $.000 * * *$ \\
\hline Físico & $\begin{array}{c}0.06 \\
(0.31)\end{array}$ & $\begin{array}{c}0.10 \\
(0.41)\end{array}$ & $\begin{array}{c}0.03 \\
(0.18)\end{array}$ & 10.727 & $.001 * * *$ \\
\hline Sexual & $\begin{array}{c}0.12 \\
(0.41)\end{array}$ & $\begin{array}{c}0.14 \\
(0.48)\end{array}$ & $\begin{array}{c}0.10 \\
(0.35)\end{array}$ & 2.093 & .148 \\
\hline Económico & $\begin{array}{c}0.04 \\
(0.25)\end{array}$ & $\begin{array}{c}0.06 \\
(0.33)\end{array}$ & $\begin{array}{c}0.02 \\
(0.16)\end{array}$ & 5.671 & $.017 *$ \\
\hline Psicológico & $\begin{array}{c}0.13 \\
(0.43)\end{array}$ & $\begin{array}{c}0.19 \\
(0.55)\end{array}$ & $\begin{array}{c}0.07 \\
(0.28)\end{array}$ & 20.205 & $.000 * * *$ \\
\hline Emocional & $\begin{array}{c}0.04 \\
(0.24)\end{array}$ & $\begin{array}{c}0.06 \\
(0.30)\end{array}$ & $\begin{array}{c}0.03 \\
(0.18)\end{array}$ & 3.539 & .060 \\
\hline Físico & $\begin{array}{c}0.04 \\
(0.20)\end{array}$ & $\begin{array}{c}0.04 \\
(0.21)\end{array}$ & $\begin{array}{c}0.04 \\
(0.20)\end{array}$ & 0.166 & .683 \\
\hline Sexual & $\begin{array}{c}0.09 \\
(0.35)\end{array}$ & $\begin{array}{c}0.15 \\
(0.46)\end{array}$ & $\begin{array}{c}0.04 \\
(0.21)\end{array}$ & 20.061 & $.000 * * *$ \\
\hline Económico & $\begin{array}{c}0.08 \\
(0.32)\end{array}$ & $\begin{array}{c}0.11 \\
(0.38)\end{array}$ & $\begin{array}{c}0.06 \\
(0.26)\end{array}$ & 4.946 & $.026^{*}$ \\
\hline Psicológico & $\begin{array}{c}0.33 \\
(0.63)\end{array}$ & $\begin{array}{c}0.38 \\
(0.68)\end{array}$ & $\begin{array}{c}0.29 \\
(0.59)\end{array}$ & 4.683 & $.031 *$ \\
\hline Emocional & $\begin{array}{c}0.04 \\
(0.23)\end{array}$ & $\begin{array}{c}0.06 \\
(0.28)\end{array}$ & $\begin{array}{c}0.03 \\
(0.17)\end{array}$ & 3.873 & $.049 *$ \\
\hline
\end{tabular}




\begin{tabular}{|c|c|c|c|c|c|c|}
\hline \multirow{2}{*}{ Maltrato informado } & \multicolumn{4}{|c|}{$\mathrm{M}(\mathrm{DE})$} & \multirow[b]{2}{*}{$\mathrm{F}$} & \multirow[b]{2}{*}{$\mathrm{p}$} \\
\hline & Tipo de maltrato & Gral. & Var. & Muj. & & \\
\hline 30. La (lo) aruñó. & Físico & $\begin{array}{c}0.10 \\
(0.37)\end{array}$ & $\begin{array}{c}0.09 \\
(0.34)\end{array}$ & $\begin{array}{c}0.12 \\
(0.39)\end{array}$ & 1.772 & .183 \\
\hline $\begin{array}{l}\text { 31. Le acarició una parte de su cuerpo de una manera vulgar, } \\
\text { sin su consentimiento. }\end{array}$ & Sexual & $\begin{array}{c}0.15 \\
(0.46)\end{array}$ & $\begin{array}{c}0.24 \\
(0.59)\end{array}$ & $\begin{array}{c}0.08 \\
(0.28)\end{array}$ & 29.782 & $.000 * * *$ \\
\hline 32. Inventó que ella (él) le había robado algo. & Psicológico & $\begin{array}{c}0.06 \\
(0.29)\end{array}$ & $\begin{array}{c}0.08 \\
(0.36)\end{array}$ & $\begin{array}{c}0.03 \\
(0.21)\end{array}$ & 7.369 & $.007 * *$ \\
\hline 33. Llegó borracho (a) a su casa a hacer escándalo. & Emocional & $\begin{array}{c}0.21 \\
(0.53)\end{array}$ & $\begin{array}{c}0.33 \\
(0.66)\end{array}$ & $\begin{array}{c}0.11 \\
(0.35)\end{array}$ & 39.245 & $.000 * * *$ \\
\hline 34. La (lo) pellizcó fuertemente. & Físico & $\begin{array}{c}0.30 \\
(0.59)\end{array}$ & $\begin{array}{c}0.27 \\
(0.58)\end{array}$ & $\begin{array}{c}0.32 \\
(0.61)\end{array}$ & 1.081 & .299 \\
\hline 35. Controló su tiempo. & Psicológico & $\begin{array}{c}0.54 \\
(0.82)\end{array}$ & $\begin{array}{c}0.48 \\
(0.78)\end{array}$ & $\begin{array}{c}0.60 \\
(0.86)\end{array}$ & 4.399 & $.036^{*}$ \\
\hline 36. La (lo) amenazó con tener una relación con otra persona. & Emocional & $\begin{array}{c}0.16 \\
(0.46)\end{array}$ & $\begin{array}{c}0.20 \\
(0.54)\end{array}$ & $\begin{array}{c}0.12 \\
(0.38)\end{array}$ & 6.306 & $.012 *$ \\
\hline $\begin{array}{l}\text { 37. La (lo) golpeó con un objeto contundente (por ejemplo, } \\
\text { un palo, un martillo, etc.). }\end{array}$ & Físico & $\begin{array}{c}0.04 \\
(0.26)\end{array}$ & $\begin{array}{c}0.06 \\
(0.32)\end{array}$ & $\begin{array}{c}0.03 \\
(0.19)\end{array}$ & 3.195 & .074 \\
\hline 38. Controló sus actividades cotidianas. & Psicológico & $\begin{array}{c}0.39 \\
(0.72)\end{array}$ & $\begin{array}{c}0.37 \\
(0.73)\end{array}$ & $\begin{array}{c}0.42 \\
(0.72)\end{array}$ & 1.071 & .301 \\
\hline $\begin{array}{l}\text { 39. La (lo) amenazó con hacerse daño a sí mismo(a), si ella } \\
\text { (él) no hacía algo que usted había dicho. }\end{array}$ & Emocional & $\begin{array}{c}0.08 \\
(0.33)\end{array}$ & $\begin{array}{c}0.11 \\
(0.39)\end{array}$ & $\begin{array}{c}0.06 \\
(0.27)\end{array}$ & 3.836 & $.050^{*}$ \\
\hline $\begin{array}{l}\text { 40. La (lo) hirió con un objeto corto punzante (por ejemplo, } \\
\text { un cuchillo, una navaja, etc.). }\end{array}$ & Físico & $\begin{array}{c}0.04 \\
(0.22)\end{array}$ & $\begin{array}{c}0.06 \\
(0.26)\end{array}$ & $\begin{array}{c}0.03 \\
(0.19)\end{array}$ & 2.622 & .106 \\
\hline 41. Le dijo que se avergonzaba de ella (él). & Psicológico & $\begin{array}{c}0.11 \\
(0.37)\end{array}$ & $\begin{array}{c}0.14 \\
(0.42)\end{array}$ & $\begin{array}{c}0.08 \\
(0.33)\end{array}$ & 5.110 & $.024 *$ \\
\hline $\begin{array}{l}\text { 42. La (lo) amenazó con encerrarla (lo) en una institución } \\
\text { mental. }\end{array}$ & Emocional & $\begin{array}{c}0.04 \\
(0.23)\end{array}$ & $\begin{array}{c}0.07 \\
(0.30)\end{array}$ & $\begin{array}{c}0.02 \\
(0.15)\end{array}$ & 8.130 & $.004 * *$ \\
\hline $\begin{array}{l}\text { 43. La (lo) quemó intencionalmente con un objeto o con una } \\
\text { sustancia (un cigarrillo, una plancha, agua caliente, etc.). }\end{array}$ & Físico & $\begin{array}{c}0.04 \\
(0.21)\end{array}$ & $\begin{array}{c}0.05 \\
(0.24)\end{array}$ & $\begin{array}{c}0.03 \\
(0.19)\end{array}$ & 1.864 & .173 \\
\hline 44. Impuso su punto de vista sobre el suyo. & Psicológico & $\begin{array}{c}0.57 \\
(0.83)\end{array}$ & $\begin{array}{c}0.61 \\
(0.86)\end{array}$ & $\begin{array}{c}0.55 \\
(0.81)\end{array}$ & 1.121 & .290 \\
\hline 45. La (lo) amenazó con matarla (lo). & Emocional & $\begin{array}{c}0.04 \\
(0.23)\end{array}$ & $\begin{array}{c}0.06 \\
(0.28)\end{array}$ & $\begin{array}{c}0.03 \\
(0.18)\end{array}$ & 2.917 & .088 \\
\hline $\begin{array}{l}\text { 46. La (lo) jaló bruscamente de alguna parte del cuerpo } \\
\text { (por ejemplo, el cabello, un brazo, etc.). }\end{array}$ & Físico & $\begin{array}{c}0.22 \\
(0.53)\end{array}$ & $\begin{array}{c}0.24 \\
(0.55)\end{array}$ & $\begin{array}{l}0.20 \\
(0.71)\end{array}$ & 1.564 & .211 \\
\hline 47. Habló mal de ella (él) con amigos o conocidos. & Psicológico & $\begin{array}{c}0.41 \\
(0.72)\end{array}$ & $\begin{array}{c}0.43 \\
(0.75)\end{array}$ & $\begin{array}{c}0.39 \\
(0.71)\end{array}$ & 0.584 & .445 \\
\hline 48. La (lo) amenazó con dañar objetos importantes para ella-él. & Emocional & $\begin{array}{c}0.06 \\
(0.28)\end{array}$ & $\begin{array}{c}0.07 \\
(0.30)\end{array}$ & $\begin{array}{c}0.06 \\
(0.26)\end{array}$ & 0.705 & .401 \\
\hline $\begin{array}{l}\text { 49. La (lo) mordió fuertemente con la intención de lasti- } \\
\text { marla (lo). }\end{array}$ & Físico & $\begin{array}{c}0.10 \\
(0.36)\end{array}$ & $\begin{array}{c}0.07 \\
(0.32)\end{array}$ & $\begin{array}{l}0.12 \\
(0.4)\end{array}$ & 3.149 & .076 \\
\hline $\begin{array}{l}\text { 50. La (lo) obligó a consumir sustancias psicoactivas (por } \\
\text { ejemplo, marihuana, cocaína, bazuco, etc.). }\end{array}$ & Psicológico & $\begin{array}{c}0.05 \\
(0.26)\end{array}$ & $\begin{array}{c}0.07 \\
(0.34)\end{array}$ & $\begin{array}{c}0.03 \\
(0.17)\end{array}$ & 4.094 & $.043 *$ \\
\hline
\end{tabular}




\begin{tabular}{|c|c|c|c|c|c|c|}
\hline \multirow{2}{*}{ Maltrato informado } & \multicolumn{4}{|c|}{$\mathrm{M}(\mathrm{DE})$} & \multirow[b]{2}{*}{$\mathrm{F}$} & \multirow[b]{2}{*}{$\mathrm{p}$} \\
\hline & Tipo de maltrato & Gral. & Var. & Muj. & & \\
\hline 51. La (lo) amenazó con matar a un familiar de ella (él). & Emocional & $\begin{array}{c}0.03 \\
(0.22)\end{array}$ & $\begin{array}{c}0.05 \\
(0.29)\end{array}$ & $\begin{array}{l}0.02 \\
(0.2)\end{array}$ & 5.168 & $.023 *$ \\
\hline 52. Intentó asfixiarla (lo). & Físico & $\begin{array}{c}0.04 \\
(0.23)\end{array}$ & $\begin{array}{c}0.05 \\
(0.25)\end{array}$ & $\begin{array}{c}0.03 \\
(0.22)\end{array}$ & 1.948 & .163 \\
\hline 53. La (lo) persiguió cuando salió de la casa o de otro sitio. & Psicológico & $\begin{array}{c}0.15 \\
(0.45)\end{array}$ & $\begin{array}{c}0.19 \\
(0.51)\end{array}$ & $\begin{array}{c}0.12 \\
(0.39)\end{array}$ & 4.678 & $.031^{*}$ \\
\hline $\begin{array}{l}\text { 54. Restringió sus movimientos amarrándola (lo), ence- } \\
\text { rrándola (lo) o por otro medio. }\end{array}$ & Físico & $\begin{array}{c}0.03 \\
(0.19)\end{array}$ & $\begin{array}{c}0.04 \\
(0.20)\end{array}$ & $\begin{array}{c}0.03 \\
(0.18)\end{array}$ & 0.329 & .566 \\
\hline 55. Mintió asegurando que ella (él) le iba a hacer daño a usted. & Psicológico & $\begin{array}{c}0.06 \\
(0.28)\end{array}$ & $\begin{array}{c}0.08 \\
(0.31)\end{array}$ & $\begin{array}{l}0.05 \\
(0.26)\end{array}$ & 3814 & .051 \\
\hline $\begin{array}{l}\text { 56. La (lo) culpó de algo malo que le había ocurrido sin } \\
\text { tener razones para ello. }\end{array}$ & Psicológico & $\begin{array}{c}0.16 \\
(0.47)\end{array}$ & $\begin{array}{c}0.19 \\
(0.52)\end{array}$ & $\begin{array}{c}0.14 \\
(0.42)\end{array}$ & 2.232 & .135 \\
\hline 57. No la (lo) dejó salir de su casa o la de ella (él). & Psicológico & $\begin{array}{c}0.10 \\
(0.37)\end{array}$ & $\begin{array}{c}0.09 \\
(0.35)\end{array}$ & $\begin{array}{c}0.10 \\
(0.39)\end{array}$ & 0.141 & .707 \\
\hline $\begin{array}{l}\text { 58. La (lo) hizo sentir ridícula(o), burlándose de ella (él) } \\
\text { frente a otras personas. }\end{array}$ & Psicológico & $\begin{array}{c}0.20 \\
(0.50)\end{array}$ & $\begin{array}{c}0.21 \\
(0.52)\end{array}$ & $\begin{array}{c}0.19 \\
(0.49)\end{array}$ & 0.281 & .610 \\
\hline 59. Le dijo que no servía para nada. & Psicológico & $\begin{array}{c}0.12 \\
(0.40)\end{array}$ & $\begin{array}{c}0.13 \\
(0.45)\end{array}$ & $\begin{array}{c}0.10 \\
(0.36)\end{array}$ & 1.301 & .254 \\
\hline 60. No la (lo) dejó entrar a la casa de ella (él). & Psicológico & $\begin{array}{c}0.11 \\
(0.39)\end{array}$ & $\begin{array}{c}0.14 \\
(0.45)\end{array}$ & $\begin{array}{c}0.08 \\
(0.34)\end{array}$ & 4.643 & $.031 *$ \\
\hline 61. Le dijo que ella (él) era desagradable para usted. & Psicológico & $\begin{array}{c}0.07 \\
(0.32)\end{array}$ & $\begin{array}{c}0.09 \\
(0.35)\end{array}$ & $\begin{array}{c}0.06 \\
(0.39)\end{array}$ & 1.550 & .213 \\
\hline 62. Le dijo que no merecía el amor de nadie. & Psicológico & $\begin{array}{c}0.12 \\
(0.42)\end{array}$ & $\begin{array}{c}0.13 \\
(0.46)\end{array}$ & $\begin{array}{c}0.12 \\
(0.39)\end{array}$ & 0.431 & .512 \\
\hline $\begin{array}{l}\text { 63. Esculcó su bolso, maletín o sus cajones sin su } \\
\text { consentimiento. }\end{array}$ & Psicológico & $\begin{array}{c}0.33 \\
(0.66)\end{array}$ & $\begin{array}{c}0.34 \\
(0.68)\end{array}$ & $\begin{array}{c}0.32 \\
(0.65)\end{array}$ & 0.144 & .704 \\
\hline $\begin{array}{l}\text { 64. La (lo) comparó negativamente con otras (os) mujeres } \\
\text { (hombres). }\end{array}$ & Psicológico & $\begin{array}{c}0.26 \\
(0.57)\end{array}$ & $\begin{array}{l}0.28 \\
(0.61)\end{array}$ & $\begin{array}{c}0.24 \\
(0.53)\end{array}$ & 0.879 & .349 \\
\hline 65. La (lo) insultó frente a otra (s) persona (s). & Psicológico & $\begin{array}{c}0.15 \\
(0.45)\end{array}$ & $\begin{array}{c}0.16 \\
(0.49)\end{array}$ & $\begin{array}{c}0.14 \\
(0.43)\end{array}$ & 0.629 & .428 \\
\hline 66. Le dijo groserías. & Psicológico & $\begin{array}{c}0.27 \\
(0.64)\end{array}$ & $\begin{array}{c}0.29 \\
(0.64)\end{array}$ & $\begin{array}{c}0.26 \\
(0.63)\end{array}$ & 0.281 & .596 \\
\hline $\begin{array}{l}\text { 67. La (lo) hizo sentirse mal, diciéndole que no lo (la) } \\
\text { satisfacía sexualmente. }\end{array}$ & Psicológico & $\begin{array}{c}0.10 \\
(0.36)\end{array}$ & $\begin{array}{l}0.13 \\
(0.41)\end{array}$ & $\begin{array}{c}0.07 \\
(0.31)\end{array}$ & 5.178 & $.023 *$ \\
\hline 68. Le prohibió que se volviera a reunir con sus amigos (as). & Psicológico & $\begin{array}{c}0.21 \\
(0.56)\end{array}$ & $\begin{array}{c}0.24 \\
(0.56)\end{array}$ & $\begin{array}{c}0.19 \\
(0.56)\end{array}$ & 1.602 & .206 \\
\hline $\begin{array}{l}\text { 69. No le permitió tener contacto con la familia de ella (él) } \\
\text { o con un miembro de la misma. }\end{array}$ & Psicológico & $\begin{array}{c}0.04 \\
(0.23)\end{array}$ & $\begin{array}{c}0.05 \\
(0.26)\end{array}$ & $\begin{array}{c}0.03 \\
(0.19)\end{array}$ & 1.271 & .260 \\
\hline 70. La (lo) acusó de ser infiel sin tener pruebas de ello. & Psicológico & $\begin{array}{c}0.51 \\
(0.75)\end{array}$ & $\begin{array}{c}0.50 \\
(0.75)\end{array}$ & $\begin{array}{c}0.52 \\
(0.76)\end{array}$ & 0.129 & .720 \\
\hline $\begin{array}{l}\text { 71. Le echó en cara lo que usted le había brindado } \\
\text { económicamente. }\end{array}$ & Psicológico & $\begin{array}{c}0.13 \\
(0.44)\end{array}$ & $\begin{array}{c}0.17 \\
(0.51)\end{array}$ & $\begin{array}{c}0.10 \\
(0.37)\end{array}$ & 5.232 & $.022 *$ \\
\hline
\end{tabular}




\begin{tabular}{|c|c|c|c|c|c|c|}
\hline \multirow{2}{*}{ Maltrato informado } & \multicolumn{6}{|c|}{$\mathrm{M}(\mathrm{DE})$} \\
\hline & Tipo de maltrato & Gral. & Var. & Muj. & $\mathrm{F}$ & $\mathrm{p}$ \\
\hline $\begin{array}{l}\text { 72. Involucró malintencionadamente a su familia o a la } \\
\text { familia de ella (él), en los conflictos de la pareja. }\end{array}$ & Psicológico & $\begin{array}{c}0.11 \\
(0.38)\end{array}$ & $\begin{array}{c}0.13 \\
(0.43)\end{array}$ & $\begin{array}{c}0.09 \\
(0.34)\end{array}$ & 2.063 & .151 \\
\hline 73. La (lo) echó de su casa. & Psicológico & $\begin{array}{c}0.13 \\
(0.43)\end{array}$ & $\begin{array}{c}0.13 \\
(0.42)\end{array}$ & $\begin{array}{c}0.14 \\
(0.43)\end{array}$ & 0.167 & .683 \\
\hline $\begin{array}{l}\text { 74. La (lo) llamó varias veces al día para averiguar que } \\
\text { estaba haciendo. }\end{array}$ & Psicológico & $\begin{array}{c}0.70 \\
(0.95)\end{array}$ & $\begin{array}{c}0.69 \\
(0.93)\end{array}$ & $\begin{array}{c}0.70 \\
(0.97)\end{array}$ & 0.011 & .917 \\
\hline 75. Utilizó el sexo para castigarla (lo) o controlarla (lo). & Psicológico & $\begin{array}{c}0.11 \\
(0.42)\end{array}$ & $\begin{array}{c}0.13 \\
(0.47)\end{array}$ & $\begin{array}{c}0.08 \\
(0.37)\end{array}$ & 3.418 & .065 \\
\hline 76. Se refirió a asuntos pasados para hacerla (lo) sentir mal. & Psicológico & $\begin{array}{c}0.47 \\
(0.81)\end{array}$ & $\begin{array}{c}0.45 \\
(0.80)\end{array}$ & $\begin{array}{c}0.49 \\
(0.83)\end{array}$ & 0.665 & .415 \\
\hline 77. Le puso sobrenombres para hacerla (lo) sentir mal. & Psicológico & $\begin{array}{c}0.14 \\
(0.46)\end{array}$ & $\begin{array}{c}0.18 \\
(0.53)\end{array}$ & $\begin{array}{c}0.10 \\
(0.38)\end{array}$ & 6.615 & $.010^{* *}$ \\
\hline 78. Le exigió obedecerle. & Psicológico & $\begin{array}{l}0.17 \\
(0.51)\end{array}$ & $\begin{array}{c}0.19 \\
(0.53)\end{array}$ & $\begin{array}{c}0.16 \\
(0.50)\end{array}$ & 1.010 & .315 \\
\hline 79. No le permitió ir al médico cuando estuvo enferma (o). & Psicológico & $\begin{array}{c}0.04 \\
(0.24)\end{array}$ & $\begin{array}{c}0.04 \\
(0.20)\end{array}$ & $\begin{array}{c}0.04 \\
(0.26)\end{array}$ & 0.116 & .733 \\
\hline $\begin{array}{l}\text { 80. La (lo) subvaloró por no haber estudiado o por no haber } \\
\text { hecho una carrera. }\end{array}$ & Psicológico & $\begin{array}{c}0.06 \\
(0.30)\end{array}$ & $\begin{array}{c}0.07 \\
(0.33)\end{array}$ & $\begin{array}{c}0.06 \\
(0.28)\end{array}$ & 0.136 & .712 \\
\hline $\begin{array}{l}\text { 81. Grabó una conversación telefónica suya sin su } \\
\text { consentimiento. }\end{array}$ & Psicológico & $\begin{array}{c}0.06 \\
(0.28)\end{array}$ & $\begin{array}{c}0.07 \\
(0.31)\end{array}$ & $\begin{array}{c}0.05 \\
(0.24)\end{array}$ & 2.482 & .115 \\
\hline $\begin{array}{l}\text { 82. Escuchó una conversación telefónica suya sin su } \\
\text { consentimiento. }\end{array}$ & Psicológico & $\begin{array}{l}0.15 \\
(0.45)\end{array}$ & $\begin{array}{c}0.16 \\
(0.46)\end{array}$ & $\begin{array}{c}0.14 \\
(0.45)\end{array}$ & 0.267 & .605 \\
\hline Total & & $\begin{array}{c}0.15 \\
(0.22)\end{array}$ & $\begin{array}{c}0.17 \\
(0.25)\end{array}$ & $\begin{array}{l}0.13 \\
(0.18)\end{array}$ & 6.659 & $.01 * *$ \\
\hline
\end{tabular}

Nota: M: Media; DE: Desviación estándar.

$* \mathrm{p} \leq .05 . * * \mathrm{p} \leq .01 . * * * \mathrm{p} \leq .001$.

Finalmente, se encontró a nivel general una correlación positiva estadísticamente significativa entre el tiempo de relación y la frecuencia de malos tratos $(r[860]=0.089$, $p=.009$ [bilateral]), así como con respecto a los seis tipos de maltrato analizados: a) Físico: $r(891)=0.872, p=.000$ (bilateral); b) psicológico: $r(857)=0.958, p=.000$ (bilateral); c) emocional: $r(884)=0.860, p=.000$ (bilateral); d) sexual $r(898)=0.772, p=.000$ (bilateral); e) económico: $r(902)=0.739, p=.000$ (bilateral) y f) negligente: $r(901)$ $=0.739, p=.000$ (bilateral).

\section{Discusión}

El objetivo general de esta investigación fue examinar la prevalencia general y por sexo de alguna conducta de maltrato de tipo físico, psicológico, emocional, sexual, económico y negligente, realizada por una muestra de adolescentes y adultos jóvenes adultos colombianos, solteros y sin hijos. Los resultados obtenidos señalan que esta forma de violencia de pareja podría afectar a una proporción muy alta de adolescentes y adultos jóvenes colombianos, tal como se encontró en los dos estudios previos ya reseñados (Rey-Anacona, 2009; Rey-Anacona, Mateus-Cubides y Bayona-Arévalo, 2010). Asimismo, indican que no existen diferencias marcadas por sexo en cuanto a la prevalencia general de malos tratos de tipo físico y psicológico, como se ha encontrado en otros estudios (e. g., Centers for Disease Control and Prevention, 2006; González y Santana, 2001; Rivera et al., 2007; Sears et al., 2007), aunque sí en los de tipo sexual los cuales parecen ser más frecuentemente ejercidos por los varones, tal como se ha hallado en otras investigaciones (e. g., Corral, 2009; Schiff y Zeira, 2005; Sears et al., 2007). Los datos también señalan que los malos 
tratos de tipo psicológico son los más frecuentes, seguidos por los físicos, los emocionales y los sexuales, tal como se ha constatado en estudios similares (Vg., Rivera et al., 2007; Sears et al., 2007), siendo menos frecuentes los malos tratos de tipo económico y negligente.

De esta manera, los datos obtenidos en esta investigación confirman que los malos tratos en el noviazgo tienden a ser menos graves que los que se han informado con respecto a la violencia marital, no solo porque fueron más frecuentes las de tipo psicológico sino porque las conductas de maltrato más comunes entre las 82 analizadas, hacían referencia básicamente a conductas de control y manipulación sobre la pareja (e. g., "La (lo) vigiló en su lugar de estudio, de trabajo o en otro espacio distinto","Controló su tiempo", "Controló sus actividades cotidianas", "Impuso su punto de vista sobre el suyo"), mientras que las conductas más graves como las agresiones físicas y las amenazas a la integridad física y psicológica fueron menos frecuentes.

Los resultados también indican que los varones tienden a ejercer más frecuentemente conductas de maltrato a su pareja que las mujeres, mostrando una frecuencia significativamente mayor no solo en la frecuencia general de los malos tratos, sino en relación con 33 de las 82 conductas examinadas, lo que señala que aunque la prevalencia de los malos tratos es similar por sexo, los varones ejercen malos tratos con una frecuencia mayor que las mujeres. De esta manera, estos datos indican la importancia de analizar aspectos de las conductas de maltrato en el noviazgo tales como la frecuencia, con el fin de comprender mejor el fenómeno, toda vez que la mayoría de los estudios tienden a centrarse en la presencia o ausencia de dichas conductas, mientras que unos pocos se han enfocado en aspectos como su intensidad, severidad, cronicidad y antecedentes contextuales (e. g., Corral, 2009; Foshee, Bauman, Linder, Rice y Wilcher, 2007: Poó y Vizcarra, 2008).

Los resultados también señalan que los malos tratos en el noviazgo se presentarían desde las primeras relaciones de pareja, es decir, aproximadamente desde los quince años de edad y que el porcentaje de adolescentes y adultos implicados en este tipo de conductas tiende a ser alto en todas las edades, si bien el tamaño de la muestra por edad es disímil tendiendo a concentrarse en el rango de 17 a 21 años de edad. Asimismo, la correlación estadísticamente significativa entre el tiempo de relación y la frecuencia promedio de malos tratos generales y con cada uno de los seis tipos de maltrato analizados, señalan que éstos tienden a incrementarse y diversificarse, afianzándose en la dinámica de la relación de pareja, por lo que la detección temprana de aquellos casos en donde han comenzado a presentarse los malos tratos, permitiría evitar que estos se incrementasen y perdurasen con el tiempo.

Los datos también confirman que en el noviazgo pueden presentarse malos tratos que pueden considerarse de tipo "económico", ya que hacen referencia a conductas como robar el dinero o bienes de la pareja, no dejarla trabajar, exigirle dinero o presionarla para cederle un bien, conductas que tradicionalmente se han considerado más comunes entre parejas casadas por los compromisos económicos implicados en este tipo de relaciones. Asimismo, reafirman que en este tipo de relaciones de pareja podrían presentarse conductas de maltrato que pueden considerarse "negligentes", por cuanto implican agredir pasivamente a la pareja al no advertirle de un peligro potencial o no protegerla o proveerle algún tipo de ayuda cuando podía hacerlo. Finalmente, los datos obtenidos sustentan la posibilidad de separar los malos tratos emocionales de los psicológicos, centrando el estudio de los primeros en conductas que generan malintencionadamente respuestas negativas de ansiedad y los segundos en conductas que tradicionalmente se han considerado de esa naturaleza como controlar a la pareja y desvalorizarla (e. g., Instituto Colombiano de Medicina Legal y Ciencias Forenses, 2005; Krug et al., 2003).

Como principales fortalezas de esta investigación se pueden señalar la gran variedad de conductas y tipos de maltrato analizados, que dan cuenta de la complejidad del fenómeno, aumentando su comprensión a través del análisis de una variable topográfica importante, como lo es la frecuencia de los malos tratos y sus diferencias por sexo. Como principales limitaciones debe indicarse el hecho de que el instrumento utilizado tiende a recoger más información sobre conductas de maltrato psicológico, físico y emocional, que de los otros tipos y que la muestra no se obtuvo a través de un procedimiento probabilístico, siendo la mayoría de los participantes de estratos socioeconómicos bajos, teniendo todos estudios universitarios, lo que da cuenta de la necesidad de realizar otros estudios de prevalencia en muestras colombianas con adolescentes y adultos colombianos con una mejor distribución de estratos socioeconómicos y niveles educativos.

\section{Referencias}

Ackard, D. M., y Neumark-Sztainer, D. (2002). Date violence and date rape among adolescents: Associations with disordered eating behaviors and psychological health. Child Abuse y Neglect, 26, 455-473.

Centers for Disease Control and Prevention (2006). Physical dating violence among High School students, United States, 2003. Morbidity and Mortality Weekly Report, 55, 532-535. 
Cornelius, T. L., y Resseguie, N. (2007). Primary and secondary prevention programs for dating violence: A review of the literature. Aggression and Violent Behavior, 12, 364-375.

Corral, S. (2009). Estudio de la violencia en el noviazgo en jóvenes universitarios/as: Cronicidad, severidad y mutualidad de las conductas violentas. Psicopatología Clínica Legal y Forense, 9, 29-48.

Foshee, V. A., Barman, K. E., Linder, F., Rice, J., y Wilcher, R. (2007). Typologies of adolescent dating violence: Identifying typologies of adolescent dating violence perpetration. Journal of Interpersonal Violence, 22, 498-519.

Gonzáles, R., y Santana, J. D. (2001). La violencia en parejas jóvenes. Psicothema, 13, 127-131.

Hanson, R. F. (2002). Adolescent dating violence: Prevalence and psychological outcomes (invited commentary). Child Abuse y Neglect, $26,447-451$

Howard, D. E., y Wang, M. Q. (2003). Risk profiles of adolescent girls who were victims of dating violence. Adolescence, 38, 1-14.

Instituto Nacional de Medicina Legal y Ciencias Forenses (2005). Reglamento técnico para el abordaje forense integral de la violencia intrafamiliar de pareja. Bogotá: Autor.

Krug, E. G., Dahlberg, L. L., Mercy, J. A., Zwi, A. B., y Lozano, R. (2003). Informe mundial sobre la violencia y la salud. Washington: Organización Mundial de la Salud.

Lewis, S. F., y Fremouw, W. (2001). Dating violence: A critical review of the literature. Clinical Psychology Review, 21, 105-127.

Matud, M. P. (2007). Dating violence and domestic violence. Spain Journal of Adolescent Health, 40, 295-297.

Ministerio de Salud de Colombia. (1993). Resolución número 008430 del 4 de octubre de 1993 "Por la cual se establecen las normas cientificas, técnicas y administrativas para la investigación en salud”. Bogotá: Autor.

Muñoz-Rivas, M. J., Graña, J. L., O’Leary, K. D., y González, M. P. (2007) Aggression in adolescent dating relationships: Prevalence, justification, and health consequences. Journal of Adolescent Health 40, 298-304.

Poó, A. M., y Vizcarra, M. B. (2008). Violencia de pareja en jóvenes universitarios. Terapia Psicológica, 26, 81-88.

Pueyo, A. A., López, S., y Álvarez, E. (2008). Valoración del riesgo de violencia contra la pareja por medio de la SARA. Papeles del Psicólogo, 29, 107-122.
Rey-Anacona, C. A. (2008a). Prevalencia, factores de riesgo y problemáticas asociadas con la violencia en el noviazgo: Una revisión de la literatura. Avances en Psicología Latinoamericana, 26, 227-241.

Rey Anacona, C. A. (2008b). Características psicológicas de adolescentes varones y adolescentes mujeres victimarios de violencia en su relación de noviazgo (informe de investigación). CIES, Universidad Pedagógica y Tecnológica de Colombia.

Rey-Anacona, C. A. (2009). Maltrato en el noviazgo de tipo físico, psicológico, emocional, sexual y económico: Un estudio exploratorio. Acta Colombiana de Psicología, 12, 27-36.

Rey-Anacona, C. A., Mateus-Cubides, A. M., y Bayona-Arévalo, P. A. (2010). Malos tratos ejercidos por adolescentes durante el noviazgo: Diferencias por sexo. Revista Mexicana de Psicología, 27, 169-181.

Rivera, L., Allen, B., Rodríguez, G., Chávez, R., y Lazcano, E. (2006). Violencia durante el noviazgo, depresión y conductas de riesgo en estudiantes femeninas (12-24 años). Salud Pública de México, 48, 288-296.

Rivera, L., Allen, B., Rodríguez, G., Chávez, R., y Lazcano, E. (2007). Prevalence and correlates of adolescent dating violence: Baseline study of a cohort of 7960 male and female Mexican public school students. Preventive Medicine, 44, 477-484.

Schiff, M., y Zeira, A. (2005). Dating violence and sexual risk behaviors in a sample of at-risk Israeli youth. Child Abuse y Neglect, 29, 1249-1263.

Sears, H. A., Byers, E. S., y Price, E. L. (2007). The co-occurrence of adolescent boys' and girls' use of psychologically, physically, and sexually abusive behaviours in their dating relationships. Journal of Adolescence, 30, 487-504.

Silverman, J. G., Raj, A., Mucci, L. A., y Hathaway, J. E. (2001). Dating violence against adolescent girls and associated substance use, unhealthy weight control, sexual risk behavior, pregnancy, and suicidality. Journal of the American Medical Association, 286, 572-579.

SPSS (2003). Statistical Package for the Social Sciences (Versión 12.0) (Software Informático). Chicago: Autor.

Stordeur, R., y Stille, R. (1989). Ending men's violence against their partners: One road to peace. Newbury Park: Sage.

Weisz, A. N., Tolman, R. M., Callahan, M. R., Saunders, D. G., y Black, B. M. (2007). Informal helpers' responses when adolescents tell them about dating violence or romantic relationship problems. Journal of Adolescence, 30, 853-868. 